\title{
The Distinctive Features of Plastic Deformation Localization in Polycrystalline Aluminum by Creep
}

\author{
Dina V. Orlova ${ }^{1,2, a)}$, Natalya V. Zarikovskaya ${ }^{3}$, Semen K. Mirgorodsky ${ }^{3}$, \\ Vladimir I. Danilov ${ }^{1,2}$, and Lev B. Zuev ${ }^{1}$ \\ ${ }^{1}$ Institute of Strength Physics and Materials Science SB RAS, Tomsk, 634055, Russia \\ ${ }^{2}$ Tomsk National Research Polytechnic University, Tomsk, 634050, Russia \\ ${ }^{3}$ Tomsk State University of Control Systems and Radio Electronics, Tomsk, 634050, Russia \\ a) Corresponding author: dvo@ispms.tsc.ru
}

\begin{abstract}
The research was performed for polycrystalline aluminum by steady-state creep. It is known that at the macroscale level, deformation exhibits localized behavior. The localization patterns would propagate over the deforming sample by steady-state creep, forming thereby a kind of phase autowave. The rate of phase autowave increases with the growing extent of straining. It is demonstrated that the processes controlling the autowave rate have the same activation volume as the processes controlling the creep rate.
\end{abstract}

Keywords: deformation macro-localization; creep; polycrystalline material

\section{INTRODUCTION}

It is now universally known that the deformation is prone to localization at all flow stages. The autowave concept generally provides a framework for discussing localized plasticity phenomena. Thus, autowave characteristics are determined by the extent of straining [1]; hence, at a given flow stage, only one kind of autowaves is likely to form. However, insufficient information is available on deformation localization in the materials tested by steady-state creep. It is an effective method providing a wealth of data on elementary deformation acts and plastic flow mechanisms. The creep rate of pure metals is entirely characterized by its dependence on stress, temperature, pressure, grain size and grain boundary state. Creep tests are usually performed at temperatures high enough to obtain a measurable creep rate; alternatively, metals having low melting point are chosen for testing by creep at room temperature. Deformation macrolocalization was investigated for polycrystalline aluminum by steady-state creep at room temperature. In choosing this material, we were also guided by the considerations of its low melting point and variable grain size.

\section{EXPERIMENTAL PROCEDURE AND RESULTS}

The tests were performed for aluminum samples having commercial purity grade $99.85 \%$. The test samples stamped out from cold-rolled sheets had been subjected to recrystallization annealing for $2.5 \mathrm{~h}$ at $670 \mathrm{~K}$. Arsenictreated material had the grain sizes of $0.03 \leq D \leq 3.20 \mathrm{~mm}$. The test samples had dog-bone shape; their work part had the dimensions of $50 \times 10 \times 2 \mathrm{~mm}$.

Uniaxial tension tests were performed on the Instron- 1185 versatile machine at $300 \mathrm{~K}$. The total deformation as a function of time $\varepsilon(t)$ was recorded for the deforming sample in situ. Using the speckle photography method, displacement vector fields were registered for the deforming sample surface; the sample elongation occurred in $100 \mu \mathrm{m}$ increments [1]. Then the plastic distortion tensor components $\left(\varepsilon_{x x}\right)$ were determined. Finally, local strain distributions were obtained for the deforming sample.

International Conference on Physical Mesomechanics of Multilevel Systems 2014

AIP Conf. Proc. 1623, 447-450 (2014); doi: 10.1063/1.4898978

(C) 2014 AIP Publishing LLC 978-0-7354-1260-6/\$30.00 


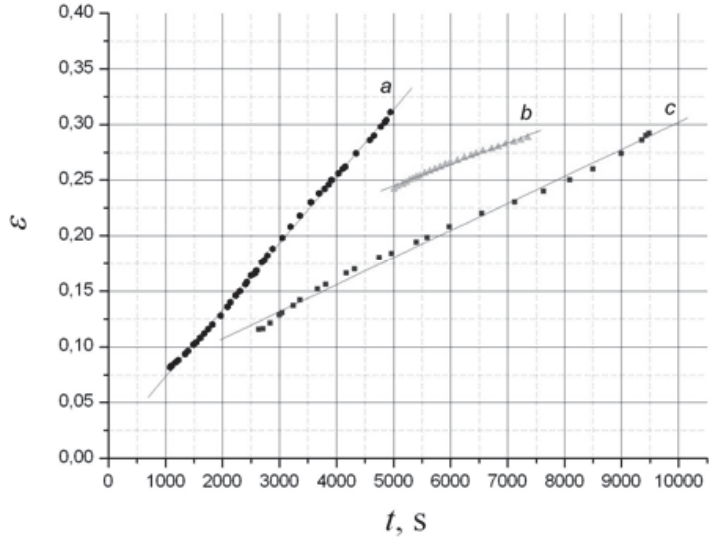

FIGURE 1. Rectilinear segments of the creep curve corresponding to the stage of steady-state creep for $\dot{\varepsilon}=$ const: 48 (a), 39 (b), $44 \mathrm{MPa}$ (c)

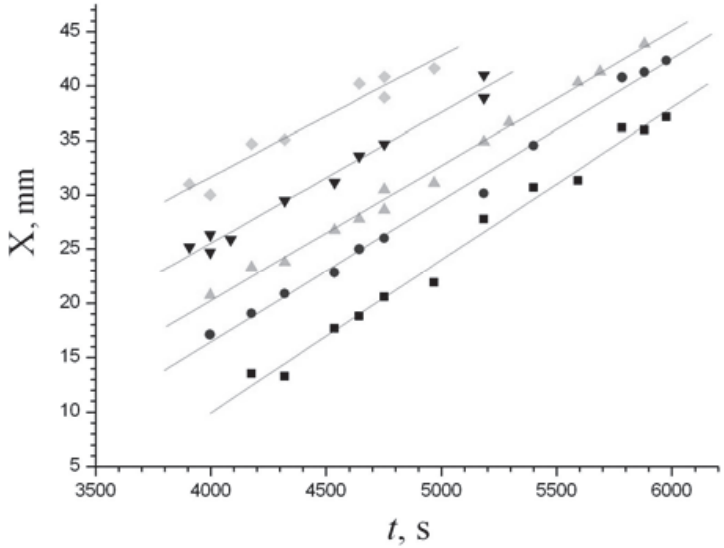

FIGURE 2. Localized deformation maxima shifting gradually with time at the linear stage of creep for $\sigma=44 \mathrm{MPa}$ (five maxima observed for the given sample are marked with different symbols)

The creep curve $\varepsilon(t)$ has three characteristic segments corresponding to the unsteady-state (exhausting) creep, the steady-state creep and the accelerated creep. This is in keeping with the accepted representations of creep [2, 3].

In what follows, the main emphasis is made on the stage of steady-state creep where the extent of straining increases with time (Fig. 1). For the latter stage, the constant creep rate could be matched against the parameters of the local strains evolution.

The local strains distributions were analyzed. It was found that the localization zones observed for the stage of steady-state creep would move concurrently along the deforming sample. The $X-t$ plots were obtained for the deforming sample (here $X$ is the localization's nucleus location and $t$ is the time). The autowave velocity ( $\left.V_{\text {aw }}\right)$ and length $\left(\lambda_{\text {aw }}\right)$ were determined from the inclinations of the plots and from the separations between the localization zones, respectively. As seen in Fig. 2, the localized deformation maxima would shift gradually with time over the sample's surface. Similar local strain patterns are observed for all the test samples. The material's grain sizes and loading conditions as well as the autowave velocity and length are presented in the Table below.

An analogy can be drawn between a set of localized deformation maxima observed for the sample subjected to steady-state creep (Fig. 2) and the pattern observed at the linear stage for the sample under active loading [1]. In the former case, however, the propagation rate of the localization maxima is lower by about one order of magnitude than in the latter case. This observation is supported by the experimental evidence obtained for fine grain aluminum and for its coarse grain counterpart tested by creep [4, 5]. It should be noted that with the increasing rate of steadystate creep, the propagation rate of localized plasticity autowaves would also increase (Fig. 3).

Thus, the autowave velocity is comparable to the creep rate, which suggests that identical specific mechanisms operate in both domains $[4,5]$. It is known that creep in metals takes place due to the thermally activated motion of dislocations [6]. The creep rate is given as

$$
\dot{\varepsilon}_{\mathrm{c}}=\dot{\varepsilon}_{0} \exp \left(-\frac{U_{\mathrm{c}}-\gamma_{\mathrm{c}} \sigma}{k T}\right)
$$

TABLE 1. Material grain sizes, testing parameters and autowave characteristics

\begin{tabular}{ccccc}
\hline$D, \mathrm{~mm}$ & $\sigma, \mathrm{MPa}$ & $\dot{\varepsilon} \cdot 10^{5}, \mathrm{~s}^{-1}$ & $V_{\mathrm{aw}} \cdot 10^{5}, \mathrm{~m} / \mathrm{s}$ & $\lambda_{\mathrm{aw}}, \mathrm{mm}$ \\
\hline 0.089 & 39.0 & $1.64 \pm 0.02$ & $0.43 \pm 0.04$ & $3.7 \pm 0.2$ \\
\hline 0.031 & 44.0 & $2.20 \pm 0.03$ & $1.24 \pm 0.10$ & $3.8 \pm 0.2$ \\
\hline 3.220 & 35.5 & $16.0 \pm 0.02$ & $2.31 \pm 0.50$ & $5.6 \pm 0.5$ \\
\hline 0.037 & 74.1 & $2.31 \pm 0.04$ & $1.55 \pm 0.08$ & $5.5 \pm 0.5$ \\
\hline 1.890 & 42.5 & $1.74 \pm 0.06$ & $0.65 \pm 0.09$ & $3.6 \pm 0.8$ \\
\hline
\end{tabular}

Footnote: $D$-grain size, $\sigma$ —stress, $\dot{\varepsilon}$ —creep rate; $V_{\text {aw }}$-autowave velocity, $\lambda_{\text {aw }}$-autowave length 


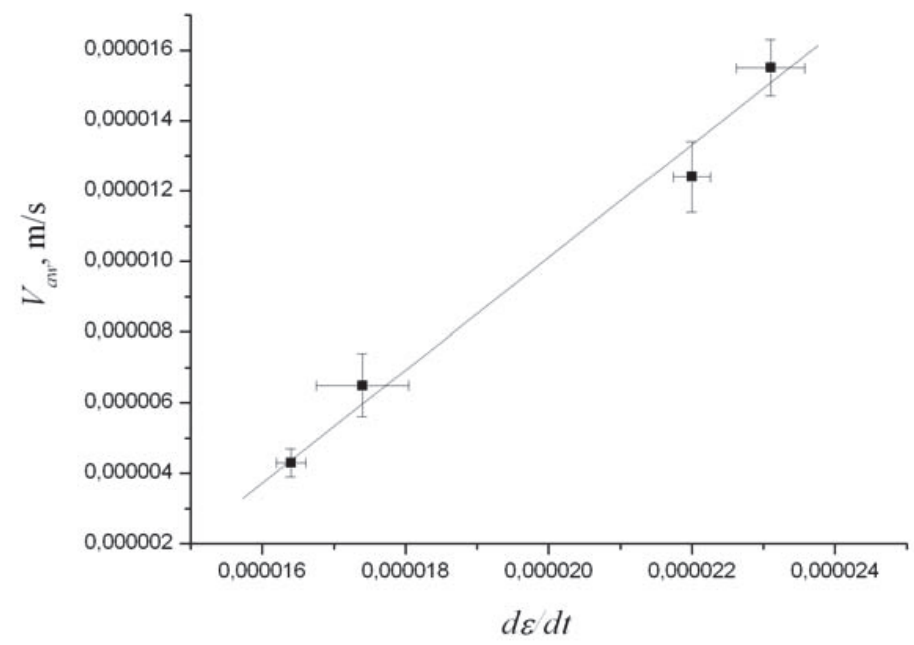

FIGURE 3. Autowave velocity as a function of steady-state creep rate

where $U_{c}$-the activation energy; $\dot{\varepsilon}_{0}=$ const; $\gamma_{c}$-the activation volume of elementary plastic flow act and $k T$ is a commonly used value. The autowave velocity is given by the following function

$$
V_{\mathrm{aw}}=V_{0} \exp \left(-\frac{U_{\mathrm{aw}}-\gamma_{\mathrm{aw}} \sigma}{k T}\right),
$$

where $V_{0}=$ const. Using the slopes of straight lines 1 and 2 (Fig. 4), the activation volumes of creep $\left(\gamma_{\mathrm{c}}\right)$ and those of autowave propagation $\left(\gamma_{\mathrm{aw}}\right)$ can be estimated as

$$
\gamma=k T \frac{\partial}{\partial \sigma}(\ln \dot{\varepsilon})
$$

Thus, it follows from (3) that $\gamma_{\mathrm{c}}=3.9 \cdot 10^{-29} \mathrm{~m}^{3}$ and $\gamma_{\mathrm{aw}}=13.6 \cdot 10^{-29} \mathrm{~m}^{3}$. Evidently, the values obtained have the same order of magnitude as those reported for fine grain $\mathrm{Al}$ and for its coarse grain counterpart $[4,5]$.

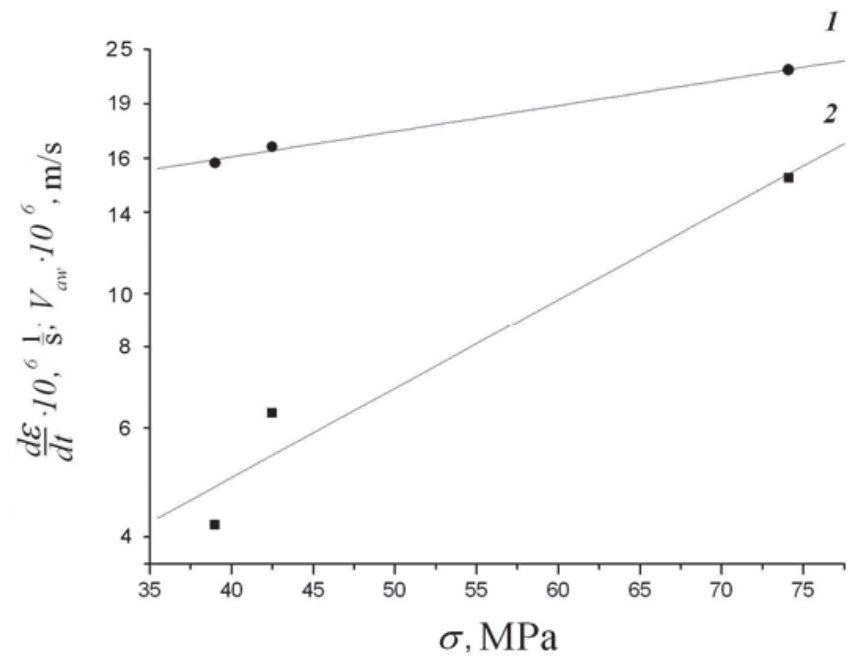

FIGURE 4. Creep rates (1) and autowave velocities (2) observed at different stress levels 
Thus, the processes in question have virtually the same activation volumes; this suggests that dislocation motion mechanisms operate in both domains. The activation volume $\gamma_{c}$ is determined by the dislocation structure of the block boundaries; specific activation processes occurring in this domain involve the motion of dislocations overcoming the barriers [7]. In this case, the rate-limiting factor for steady-state creep is the velocity of dislocation motion. Consider now the autowave velocity observed for the linear work hardening stage caused by active loading: the $V_{\text {aw }}$ value is determined by the ratio of densities of immobile to mobile dislocations [1]. With the increasing density of accumulated defects, the energy of immobile dislocations increases and the $V_{\text {aw }}$ value decreases. Due to the dislocation motion, a greater quantity of energy is dissipated to heat to cause an increase in the $V_{\text {aw }}$ value.

\section{CONCLUSIONS}

The similarity of the macrolocalization pattern observed for the stage of steady-state creep and that of the autowave pattern (emergent at the stage of linear work hardening caused by active loading) suggests that the both have the autowave nature.

A correlation is found to exist between the activation volumes of creep rates on one hand and the activation volumes of localized plasticity autowaves on the other hand. This suggests that in both cases, at least one of the controlling processes is the dislocation motion.

The work was supported partially by the Russian Foundation for Basic Research, grant No. 14-08-00299.

\section{REFERENCES}

1. L. B. Zuev, Phys. Wave Phenomena. 3, 166 (2012).

2. V. M. Rosenberg, Creep in Metal (Metallurgy, Moscow, 1967).

3. A. J. Kennedy, Creep and Fatigue in Metals (Metallurgy, Moscow, 1965).

4. V .I. Danilov, A. A. Yavorsky, L. B. Zuev, and V. E. Panin, Izv. Vuzov. Phys. 4, 5 (1991).

5. V. I. Danilov, S. V. Konovalov, S. V. Zhuravleva, L. B. Zuev, and V. E. Gromov, Zurn. Tekhn. Fiz. 3, 92 (2005).

6. D. Kuhlmann-Wilsdorf, Dislocation in Solids (Elsevier, Amsterdam, 2002).

7. J. J. Gilman, Mech. Mater., 17, 83 (1994). 